\title{
CHARAKTERISTIK DER EINARTENHONIGE
}

\author{
\%. DEMIANOWICZ \\ Instytut Sadownictwa, Oddsial Pszcselnictwa \\ Skiernievice R. Warszai' $\}^{\prime}$ \\ Pologne
}

Die Pollenanalyse des Honigs beruht auf der Annahme, dass die Bienen bei der Nektarsammlung auch eine gewisse Menge des Pollens der betreffenden Pflanze mitnehmen. Der mitgenommene Pollen bleibt weiter im Honig, der aus dem Nektar entsteht.

Die Anwesenheit von Pflanzenteilen erlaubt im weiten Masse die Herkunft des Honigs zu beurteilen. Diese Analyse ist auch für die Imkerpraxis von Bedeutung, besonders bei der Beurteilung des Werts verschiedener Trachtpflanzen.

Die Pollenanalyse in ihrer bisher durchgeführten Form ist eigentlich eine qualitative Analyse, da der "Einstäubungsgrad " des Honigs von verschiedenen Pflanzen sehr unterschiedlich ist. Infolgedessen entspricht bei den Mischhonigen das Mengenverhältnis der Pollenarten nicht dem Mengenverhältnis der Nektararten, die zur Entstehung des Honigs beigetragen haben. Es besteht trotzdem immer die Tendenz, beide Mengenverhältnisse für identisch zı halten. Das führt zur Überschätzung einzelner Honigpflanzen (z. B. Myosotis silvatica) oder zur Infragestellung des Trachtwertes anderer Pflanzen (z. B. Tilia cordata).

In der Bienenbotanik gehört die Frage nach dem Trachtwert der Linde zu den höchst umstrittenen. Obwohl diese Frage viele Autoren in verschiedenen Ländern Europas (Zander, Koch, Maurizio, Hasi,issie, de Boer, Martimo) beschäftigt hat, erwähnt ZANDER nur ca. 20 Nord-und Mitteleuropäische Honige, in denen der Lindenpollen als Leitpollen auftritt, Koch Io und MaUrizio ebenfalls Io. In den anderen Proben tritt er als Begleitpollen oder Einzelpollen auf. Aus diesem Grunde sind viele Forscher zu der Ansicht gekommen, dass die Linde als Trachtpflanze nur geringen Wert besizt, was einerseits durch eine geringe Nektarproduktion, andererseits durch die relative Seltenheit der Linde erklärt wurde. Qualitative Untersuchungen von A. Maurizio, zeigen, dass die Mehrzahl der Lindenhonige verhältnismässig pollenarm ist; infolgedessen setzte sie die Grenze des Leitpollens für Linde au 35-40 p. Ioo herab.

In der Sowjetunion and in Rumänien dagegen wird die Linde von den Imkern als eine der hervorragensten Honigpflanzen geschätzt, besonders dort, wo sie in günstigen Klimaverhältnissen wächst. In diesen Ländern gibt sie in guten Jahren pro Tag und Volk $5,5 \mathrm{~kg}$ Honig und mehr. 
In Polen wird die Linde von den Imkern ebenfalls hoch geschätzt. Das wurde durch die Rundfrage der Imkerabteilung des Wissenchaftlichen Staatsinstituts für Landwirtschaft im Jahre 1937 bestätigt. Auf 683 Fragebogen wurde die Linde $575 \mathrm{mal}$ als Trachtpflanze erwähnt, $283 \mathrm{mal}$ wurde ihr eine erstklassige Bedeutung beigemessen. In den übrigen 292 Fällen wurde sie als Bienenweidepflanze zweiter Ordnung bezeichnet. A. Demixowicz hat auf Grund von Wagstockbeobachtungen in Pulawy den maximalen Honigertrag der Linde auf $5,5 \mathrm{~kg}$ pro Tag und Volk ermittelt. Auf Grund der Tageszunahmen, die an einem Versuchsvolk im Jahre I937 vor und nach dem Aufblïhen der Winterlinde gemessen wurden, konnte festgestellt werden, dass der Honig im entsprechenden Zeitabschnitt zu mindestens 80 p. IoO von der Linde stammte. Der gleiche Honig wurde K. LuBLINER-MIANOwska zur Untersuchung übergeben. Auf Grund der Pollenanalyse bezeichnete sie ihn als einen Rapshonig mit einem unbedeutenden Lindenanteil. Diese Firmittlung kann leider nicht mehr mit Zahlen belegt werden, da die Protokolle während des Krieges verloren gegangen sind.

Der krasse Gegensatz zwischen diesen Beobachtungen und den Ergebnissen der bisherigen Pollenanalyse führte zu der Annahme, dass der Einstäubungsgrad des Nektars bei den Pflanzen sehr verschieden ist. Las zwang uns, eine bessere Grundlage zur Auswertung der Pollenanalyse zu suchen und eine neue exaktere Methode auszuarbeiten.

Unserer Ansicht nach muss man zuverlässige Koeffizienten für die einzelnen Pollenarten ermitteln. Diese können gewonnen werden durch die Auszählung der Pollenkörner in I g eines Honigs, der ausschliesslich von einer Pflanzenart eingetragen wurde. Mit diesen Koeffizienten wird man eine bessere quantitative Schätzung der einzelnen Trachtanteile in Mischhonigen durchführen können.

\section{ME'THODIK}

Schon im Jahre 1951 haben wir den ersten Versuch gemacht, solche Honige von nur einer Ptlanzenart ("Einartenhonige ") zu gewinnen. Wir zwangen die Bienen, den Nektar von nur einer Trachtpflanze in einem abgeschlossenen Raum zu sammeln. Zu diesem Zwecke bedienten wir uns zusammenlegbarer Flugkäfige. Zuerst isolierten wir auf diese Weise eine Senfparzelle von $64 \mathrm{~m}^{2}$. In späteren Versuchen begnügten wir uns mit Parzellen von $48 \mathrm{~m}^{2}$, schliesslich mit Flächen von nur $36 \mathrm{~m}^{2}$. Im Falle von Bäumen, wie Tilia cordata, Robinia pseudacacia und Malus domestica isolierten wir mit Hilfe von Gaze einen Raum von $60-80 \mathrm{~m}^{3}$. Unter den Flugkäfig stellten wir ein kleines, mit einer Schutzhaube versehenes Zandersches Begattungskästchen, wie es üblicherweise zur Königinnenzucht verwendet wird. Später setzten wir ein Einwabenkästchen in ein normales Bienenvolk hinein (zur Warmhaltung des Versuchsö̈lkchens). Das Einwabenkästchen erhielt ein Flugloch in den Flug. käfig hinein. Das Versuchsvölkchen soll nicht gross sein (300-100 Bienen), da bei einer stärkeren Besetzung die zur Verfügung stehende Bienenweide den Ernährungsbedürfnissen des Völkchens nicht genügen würde. Die besten Ergebnisse werden damn erzielt, wenn man etwa 10-20 Tage alte Bienen verwendet, die ihre Flugtätigkeit erst im Flugzelt entfalten. Die älteren Bienen gewöhnen sich nur schlecht an die Arbeit in einem isolierten Raum, die meisten von ihnen stossen sich an den Netzwänden des Käfigs zu Tode.

Wie unsere Beobachtungen zeigten, passen sich die jüngeren Bienen der Arbeit im geschlossenen Raum leicht an und sammeln nicht nur Nektar sondern auch Pollen.

Die Kästchen mit den Versuchsvölkchen sollen einen oder mehrere Tage eingeschlossen bleiben, damit die wenigen Flugbienen, die sich immer noch darin finden, Zeit haben, die Lage des Mutterstocks und die Pflanze, die sie zuletzt beflogen haben, zu vergessen. Die so vorbereiteten Bienen führen den Orientierungsflug nach dem Öffnen besser aus. Anfangs gaben wir den Völkchen eine Wabe mit 
Io bis 20 gedeckelten und einigen offenen Brutzellen, in den späteren Versuchen eine leere Wabe, ferner eine befruchtete oder cine unbefruchtete Königin, damit die Bienen in den Kästchen normal arbeiten. Die Königin soll gekäfigt sein, damit sie, falls sie befruchtet ist, nicht durch Bruteinschlag den IIonigverbrauch vergrössert, oder, falls sie unbefruchtet ist, nicht bei Ausflugsversuchen verloren geht. Die Hauptschwierigkeit der Methode beruht darauf, dass vor Versuchsbeginn und während schlechten Wetters die Bienen mit sirup gefüttert werden müssen. Fs besteht die Gefahr, dass ein 'Teil des Sirups in den Waben deponiert wird, auch wenn die Dosen so bemessen werden, dass sie gerade den Tagesbedarf decken. Wenn die Tracht aus der Versuchsparzelle einsetzt, muss der 'Juckerfütterungshonig eliminiert werden. Dazu wird in den Morgenstunden bei günstigem Wetter der ganze I Ionig herausgenommen. Da aber ein Teil davon von den Bienen in die Honigblase aufgenommen wird, muss das Verfahren am nächsten Tag wiederholt werden. (Um die Futteraufnahme in die IIonigblase möglichst niedrig halten, soll man bei der Arbeit keinen Rauch verwenden).

Ich möchte annehmen, dass nach zweimaliger Durchführung des Vingriffs der Sirupgehalt sehr klein und der dadurch entstehende Fehler unbedeutend sein wird. In vielen späteren Versuchen wurde die Wabe mit denn siruphaltigen Mischhonig heraungenonmen und eine satubere leere Wabe eingehängt.

Je reifer der gesammalte Honig ist, desto besser wird er für die Analyse sein. Zuerst wurden die Honigproben während der Blütezeit der gegebenen Pflanzenart mehrere Male mit (ilaspipetten, spätter mit IIilfe eines Exhaustors entnommen und in Probiergläschen zusammengegossen. Da es vorkommen kann, das; die Bienen Honig in Zellen deponieren, in die vorher Pollen eingetragen wurde, ist es besser, sich mehrerer l'ipetten und mehrerer Probiergläschen zu bedienen. Das erlaubt später offensichtlich sekundär eingestäubte I Ionigproben auszuscheiden

Nach dem Abblühen der Versuchsparzelle wird die Wabe aus dem Finwabenküstchen unter Zuhilfenahme einer Einlage geschleudert. Di: Einlage ist aus Blech gefertigt und besteht aus cinem oberen quadratischen Teil, der di: Rähmchen oder Wabenstücke aufnimmt. Drahtgeflecht sorgt dafür, dass die Wabe nicht unmittelbar den Blechwänden aufliegt. I)er quadratische Oberteil der Einlage setzt sich nach unten in einen dreieckigen abgeflachten Trichter fort, in dem sich der ausgeschleuderte Honir sammelt. Durch eine Öffnung an der Spitze kann der Honig abgezapft werden. An der Aussenseite der Einlage betinden sich Haken, die das Einhängen in jede beliebige Honigschleuder erlauben. İm eine sekundäre Jinstäubung mit Pollen zu verhindern, werden alle Pollenzellen vor dem Schleudern mit flüssigem Wachs plombiert. In den Fällen, in denen man keinen reifen Honig erlangen konnte, dickte man den Honig in ein

Der ganze Honig wurde zusammengegosien, gründlich vermengt und aus ihm mindestens 4 Proben für die mikroskopische Intersuchung entuommen. Die Pollenkörner wurden unmittelbar in Honig (im Falle von Mlyosotis in Honiglösung) ge zählt. Wir legten ein Tröpfchen auf ein Deckgläschen bekannten (iewichts und wogen anschliessend wieder. Die Ilonigmenge soll so klein sein, dass nach dem Iimkehren des Deckgläaschens und clem Aulegen auf den Objektträger der IIonig nicht seitlich über die Ränder hinausquillt. Die Ilonigmenge überschreitet im allgemeinen nicht $20 \mathrm{mg}$.

Die Zählung der Pollenkörner erfolgte unter dem Mikroskop mit Ililfe eines Kreuztisches und eincm Okularnetzmikrometers. Die Zahl der Pollenkömer wurde für ein Gramm Honig berechnet.

Die Farben der Einartenhonige wurden nach den Farbtafeln von A. Maer\%, und II. R. Paul, A dictionary of color, lew York 19,30 , bestimmt.

\section{VERSUCHSERGEBNISSE}

Im Laufe von I3 Jahren (von I95I-I963) wurden Einartenhonige von 46 Pflanzenarten gewonnen. Da die Honige einiger Arten 2-3 Jahre wiederholt gewonnen wurden, beträgt die gesamte Anzahl der Honige 6o. Wir haben bis jetzt über diese Honige 7 Arbeiten veröffentlicht.

Die vorliegende Publikation fasst die Eirgebnisse zusammen, die an allen 46 Honigen gewonnen wurden, dabei werden i6 Honige zum erstem Mal beschrieben.

Die gewonnenen Honige gehören zu 22 Pflanzenfamilien. Die Labiatae sind mit 7 Arten vertreten, die Compositae gleichfalls mit 7 Arten, die Boraginaceae und die Papilionaceae mit 5, die Umbelliferae, Cruciferae, Rosaceae und Scrophulariaceae mit je 2 Arten; die übrigen Familien nur mit je einer Art (tab. I). 
Familie und Art.

Famille et espèce
Jahr

Anné
Anzahl der Gewomnene Pollenkürner Honigmenge je $10 \mathrm{~g}$ Honig (in g)

Nombre de Quantité de grains de pollen miel récolté par $10 \mathrm{gde}$ miel (en $r$ )

\section{Jabiatide}

1) Salvia nemorosa $L \ldots \ldots \ldots \ldots \ldots \ldots \ldots$

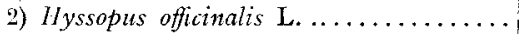

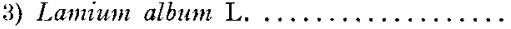

4) Dracocephalum moldavicum L......... Dracocephalum moldavicum L.........

5) Salvia officinalis $\mathbf{L} \ldots \ldots \ldots \ldots \ldots \ldots$

6) Leonorus cardiaca var. villosus DESF.....

7) Marrubium attlgare L.
II Compositale

1) Centaurea jacea L. . . . . . . . . . . . 1963

2) Echinops commutatus JURATZ........ 1963

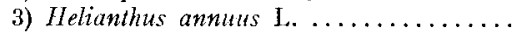

4) Centaurea cynanus L..............

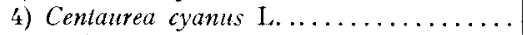

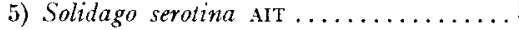

6) Taraxacum officinale $\mathrm{WE \textrm {B }} \ldots \ldots \ldots \ldots \ldots$

7) Helenium autumale $\mathrm{L}$.

III Papilionaceae

1) Robinia pseudacacia L.............

2) Onobrychis viciaefolia scop ...........

3) Trifolium repens $\mathrm{L} . \ldots \ldots \ldots \ldots \ldots \ldots$ Trifolium repens $\mathrm{L} \ldots \ldots \ldots \ldots \ldots \ldots \ldots$ Trifolium repens $\mathrm{L} \ldots \ldots \ldots \ldots \ldots \ldots$

1) Melilotus albus $\mathrm{NED} \ldots \ldots \ldots \ldots \ldots \ldots$

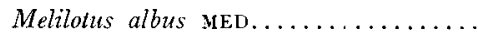

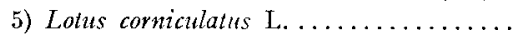
IV Boraginaceae

1) Borago officinalis I

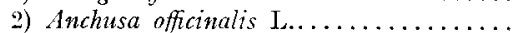

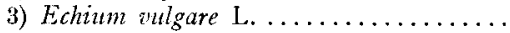

4) Cynoglossum officinale L..

5) Myosotis sitzatica Hoffy.............

$\mathrm{V}$ Hydrophyllaceae

1) Phacelia tanacaelifolia BENTH Phacelia tanacaetifolia BENTH.

\section{Umbelliferae}

1) Coriandrum satioum L.

2) Archangelica officinalis HOF II

\section{Cruciferae}

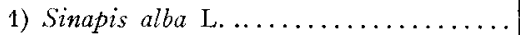

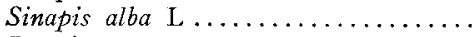

2) Brassica napus var. oleifera METZGER..

\begin{tabular}{l}
\hline \\
1961 \\
1960 \\
1963 \\
1959 \\
1958 \\
1961 \\
1960 \\
1961 \\
-19 \\
1963 \\
1963 \\
1961 \\
$195 \mathrm{z}$ \\
1958 \\
1963 \\
1963 \\
1963
\end{tabular}

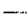

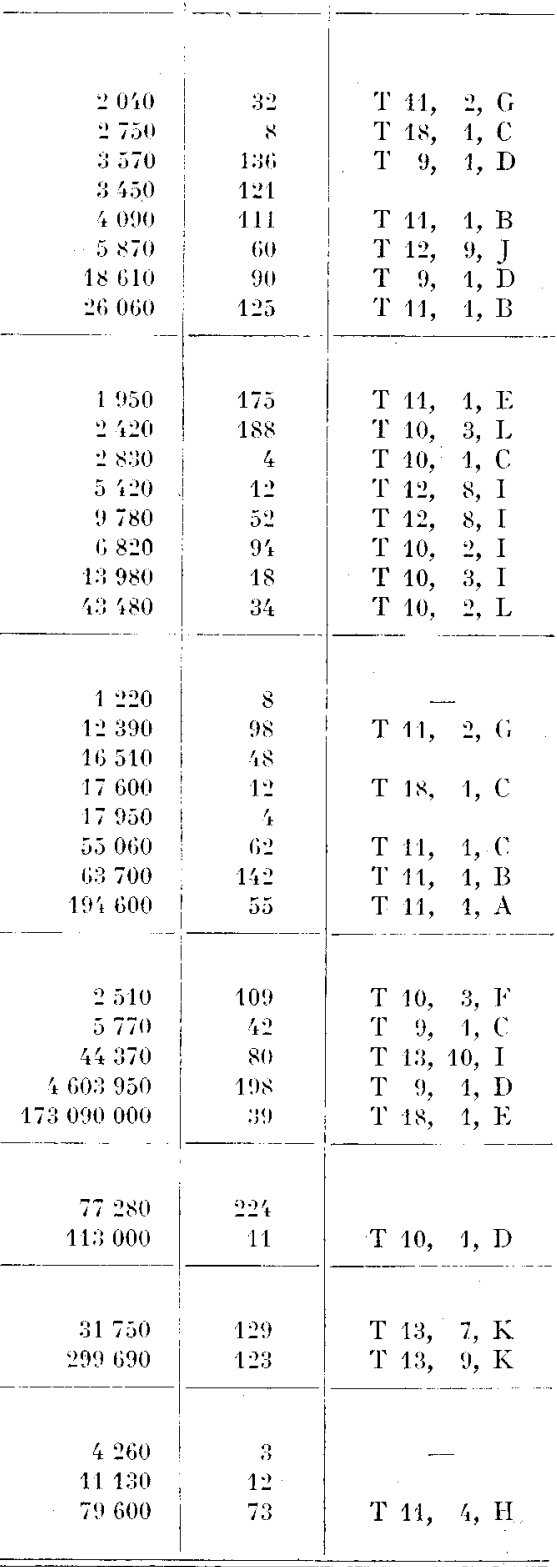

Honigfarbe Couleur du miel 
TABLEAU I TABELI,E I (Folge)

\begin{tabular}{|c|c|c|c|c|}
\hline $\begin{array}{l}\text { Familie und Art. } \\
\text { Famille et espece }\end{array}$ & $\begin{array}{l}\text { Jahr } \\
\text { Année }\end{array}$ & $\mid \begin{array}{c}\text { Anzahl der } \\
\text { Pollenkörner } \\
\text { je } 10 \text { g Honig } \\
\text { Nombre de } \\
\text { Mrains de jollen } \\
\text { prir } 10 \text { g de miel }\end{array}$ & $\mid \begin{array}{l}\text { (Cewonnene: } \\
\text { lonigmenge } \\
\text { (in g) } \\
\text { Quantité de } \\
\text { miel récolté } \\
\text { en }\end{array}$ & $\begin{array}{l}\text { Honigfarbe } \\
\text { Couleur du miel }\end{array}$ \\
\hline VIII Rosaceae & & & & \\
\hline 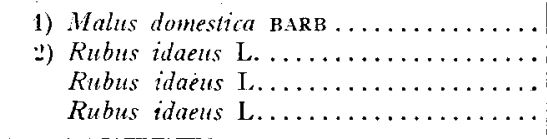 & $\begin{array}{l}1956 \\
19515 \\
1957 \\
1958\end{array}$ & $\begin{array}{l}19: 290 \\
88020 \\
56150 \\
97 \div 00\end{array}$ & $\begin{array}{l}19 \\
80 \\
50 \\
60\end{array}$ & $\begin{array}{l}\text { T } 10, \text { i. L } \\
\text { T } 12,7, \mathrm{~L}\end{array}$ \\
\hline $\begin{array}{l}\text { IX Saxifragaceae } \\
\text { 1) Ribes anlgare LAsI......... } \\
\text { X Ascepiadareate } \\
\text { 1) Asclepias syriaca L. ....... }\end{array}$ & 1967 & 81.51 & $: 350$ & T $10,1,1$, \\
\hline $\begin{array}{l}\text { XI (Enotheraceue } \\
\text { 1) Chamaenerion angustifolium L... } \\
\text { Chamaenerion angustifolium } \mathrm{L} \text {... }\end{array}$ & $\begin{array}{l}1961 \\
196: 3\end{array}$ & $\begin{array}{r}6600 \\
16 \geqslant 0\end{array}$ & $5 !$ & $\begin{array}{l}\mathrm{T} 11, \quad 2, \\
\mathrm{~T} 10,1, \mathrm{C}\end{array}$ \\
\hline 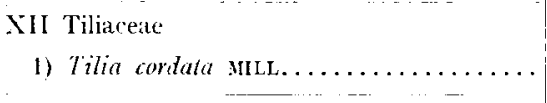 & 1951 & 18600 & 15 & T $10,:$ i \\
\hline $\begin{array}{l}\text { XIll Scrophulariaceae } \\
\text { 1) Scrophularia nodosa } \mathrm{L} . \ldots \ldots \ldots \ldots \ldots \\
\text { 2) Digitalis purpurea } \mathrm{L} . \ldots \ldots \ldots \ldots \ldots\end{array}$ & $\begin{array}{l}1966) \\
1959 ?\end{array}$ & $\begin{array}{rl}2 & 270 \\
18 & =010\end{array}$ & $\begin{array}{r}40 \\
112 \\
\end{array}$ & $\begin{array}{l}\mathrm{T} \quad 13,10, \mathrm{~L} \\
\mathrm{~T} \quad 9,1, \mathrm{l}\end{array}$ \\
\hline $\begin{array}{l}\text { XIV Jiliaceate } \\
\text { 1) Allium cepa } \mathrm{I} . \ldots . .\end{array}$ & 1938 & $10 \times 70$ & 11 & --- \\
\hline $\begin{array}{l}X V \text { Polemoniaceae } \\
\text { 1) Polemonium coeruleum } 1 . \ldots .\end{array}$ & 1958 & 6660 & 135 & $\mathrm{~T} 10.7, \mathrm{~L}$ \\
\hline $\begin{array}{l}\text { XVI Geraniaceat } \\
\text { 1) Geranium pratense I. }\end{array}$ & $196: 3$ & 1.1420 & $9=$ & $\mathrm{T} 10, \quad 1, \mathrm{c}$ \\
\hline $\begin{array}{l}\text { XVII Rutaceae } \\
\text { 1) Ruta graveolens I..... }\end{array}$ & $196: 1$ & $1 ; 0 \div 0$ & 297 & $\mathrm{~T} 10,5, \mathrm{~K}$ \\
\hline $\begin{array}{l}\text { XVII Polygonaceae } \\
\text { 1) Fagopyrum sagittatum GIIIs.......... }\end{array}$ & $1963: 3$ & 58010 & $1^{\prime} t^{\prime}$ & $116,12, \mathrm{~A}$ \\
\hline $\begin{array}{l}\text { XIX Resedaceat } \\
\text { 1) Reseda lutea 1. u. R. luteola L........ }\end{array}$ & $195 !$ & 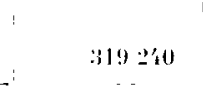 & 69 & T $1: 6$, II \\
\hline $\begin{array}{l}\text { XX Lythraceac } \\
\text { 1) Lythrtm salicaria } \mathrm{L} . \ldots \ldots\end{array}$ & $196:$ & 177090 & 138 & $T 12, \quad \therefore$, \\
\hline $\begin{array}{l}\text { XXI Cucurbitacede } \\
\text { 1). Cucumis sativus L.... }\end{array}$ & $1961 \mid$ & 860 & ? & $\mathrm{T} 10,: ;, \mathrm{I}$ \\
\hline $\begin{array}{l}\text { XXII Malvaceae } \\
\text { 1) Althaea officinalis } \mathrm{L} . \ldots \ldots \ldots \ldots\end{array}$ & 1960 & 2.50 & ‘? & T $\quad 9, \quad 2, \quad I 1$ \\
\hline
\end{tabular}


Bis jetzt wurden 70 Versuche durchgefürht .Zehn von den Versuchen sind missgliickt und das hauptsächlich infolge ungüngstiger Wetterverhältnisse, unzulänglicher Nektarbildung (z. B. bei Ornithopus sativus) oder auch infolge Nichtbefliegens der Blitien durch die Bienen (z. B. bei Nicotiana rustica). Im letzteren Fall sammelten die Bienen nur solange Nektar aus den Blüten, solange Sirup im Bienenstock vorhanden war. Nach dem Verzehren des Sirups gaben die Bienen die Nektarsuche auf, obwohl das Wetter sehr günstig und die Nektarproduktion der Bliuten reichlich war. I)ie Menge des gewonnenen Honigs schwankte zwischen einigen Gramm bei Cucumis satizus, Helianthus annums und Robinia pseudacacia bis $\approx u 350 \mathrm{~g}$ bei Asclepias syriaca. Grosse Mengen gewann man aus Ruta graveolens (297 g), Phacelia tanacaetifolia (224g), Cynoglossum officinale ( $\mathrm{Ig} 6 \mathrm{~g}$ ) und Echinops commutatus ( $1 \mathrm{I} 8 \mathrm{~g}$ ).

Was die Menge und Vielzahl der gewonnenen Honigproben anbetrifft, so war das Jahr rg63 mit seinem heissen und trockenen Sommer ein Rekordjahr.

Ausgenommen Digitalis purpurea, Geranium pratense, Marrubium vulgare und Vicotiana rustica waren in den Waben immer Pollenzellen zu finden. Beim Experiment mit Asclepias syriaca befanden sich die Pollinien, die den Bienen an den Beinchen haften blieben, am Boden des Bienenstocks, vereinzelt aber auch in den Zellen.

Die Mehrzahl der Iinartenhonige kristallisierte sehr gut. Eine Ausnahme bildete der Honig aus Robinia pseudacaccia und der von Digitalis purpurea, der seit 1959 fliussig geblieben ist. Ton den Honigen des letzten Jahres blieben flüssig die Proben von Lamium album, Ruta grateolens und Anchusa officinalis.

Schwach kristallisierten Honige, die aus Polemonium coeruleum und Chamaenerion angustifolium gewonnen wurden. Fine flïssige Schicht an der Oberfläche bildete sich bei Honigen von Centaurea cyanus und Myosotis silvatica.

Sehr schnell kristallisierte Honige aus Brassica napus var. oleifera, Taraxacum officinale und Solidago serotina. Was die Farbe anbetrifft, so iiberwiegen helle oder farblose Honige. Die dunkelsten línartenhonige waren die von Fagopyrum saggitatum, Coriandrum sativum, Archangelica officinalis, Lythrum salicaria. Die Mehrzahl der Compositenhonige zeichnet sich durch eine hellgelbe Farbe aus.

Der Einstäubungsgrad bei den Einartenhonigen ist sehr unterschiedlich; die Zahl der Pollenkörner in je Io g Honig schwankt zwischen o bei Asclepias syriaca und 173090 ooo bei Myosotis silvatica. Diese grossen Unterschiede treten nicht nur bei Arten auf, die verschiedenen Familien angehören, sondern auch bei Arten innerhalb einer Gattung. So beträgt z. B. der Einstäubungsgrad des Honigs aus Centaurea jacea I $95^{\circ}$ Pollenkörner pro Io g; im Honig aus Centaurea cyanus finden sich 5420 u. 9780 Pollenkörner pro Io g, also drei bis fünf mal mehr. Grosse Unterschiede treten innerhalb der Familie der Labiatae auf, wo man bei Salvia nemorosa in Io $g$ Honig 2 o4o Pollenkörner findet und bei Salvia officinalis beinahe dreimal mehr (5870), bei Leonorus cardiaca var. villosus ist die Zahl der Pollenkörner ca. neunmal grösser als bei Saliia nemorosa (I86Io), bei Marrubium vulgare ca. zwölfmal grösser (26 o60). Marrubium ist eine Art, die sehr kleine Bliiten hat, die sich durch eine kurze und schmale Kronröhre auszeichnen, in der 4 Staubblätter und I Fruchtknoten verborgen sind. Die Biene, welche solch eine Bliite befliegt, stösst den Pollen in den Nektar. Noch grössere Unterschiede des Einstäubungsgrads sind innerhalb der Compositae zu verzeichnen : von I $95^{\circ}$ bei Centaurea jacea bis zu 43840 bei Helenium autumnale. Taraxacum officinale nimmt einen mittleren Platz ein ( 13980 Pollenkörner pro ro $\mathrm{g}$ 
Honig). Der hohe Einstäubungsgrad des Honigs von Helenium autumnale ist bedingt durch die grosse Pollenmenge, die in diesen Blïten gebildet wird. Die Nektarsammlerinnen waren gänzlich eingepudert mit Pollen und uibertragen mit ihren Beinen und dem Haarkleid den Pollen auf die Wabe des Versuchskästchens.

Die grössten Unterschiede finden sich jedoch bei den Honigproben aus verschie denen Arten aus der Familie der Boraginaceae. I)ie Zahl der Pollenkörner pro Io g Honig liegt im Falle von Borago officinalis bei 2150 bei Myosotis silvatica bei I73 090 000. Hier macht sich der Finfluss von zwei Faktoren bemerkbar : der Bau der Blïten und die Grösse der Pollenkörner. Der Nektar ist bei Borago tief in der Bliite versteckt und die Möglichkeit der Beimengung von Pollenkörnern ist gering. Echium vulgare hat weitgeöffnete Bliiten, so dass der Pollen leicht in den Nektar fällt.

Die Blïten von Anchusa officinalis und von Cynoglossum officinale sind gleichartig gebaut, aber die Grösse der Pollenkörner ist verschieden : bei Anchusa beträgt sie 36,8:27 $\mu$, bei Cynoglossum officinale dagegen 8,7: $4 \mu$ Myosotis silvatica hat sehr kleine Blüten und kleine Pollenkörner $(6: 2,5 \mu)$. Sowohl bei Anchusa wie auch. bei Cynoglossum gelangt durch die Tätigkeit der Bienen ein Teil des Pollens aus den Antheren in den Nektar. In Falle von Myosotis stossen die Bienen fast die gesamte Pollenmenge in den Nektar.

Die Tätigkeit des Ventiltrichters in der Honigblase der Sammlerinnen wird durch grosse Pollenkörner mehr angeregt als durch kleine. I)ies führt dazu, dass der Nektar von Myosotis and Cynoglossum in der Honigblase der Biene nur wenig ron Pollen gereinigt wird und ein Honig mit hohem Pollengehalt entsteht. Der Honig von Cynoglossum officinale enthält 4603950 Pollenkörner in Io g, der Honig von Myosotis sogar I73 090 ooo. I iese Zahlen beweisen, daß die häufig in der Fachliteratur angeführten Myosotishonige nur zu einem sehr geringen Anteil aus Myosotis-NektaI entstanden sind. Der höchste Anteil dieser sogenannten Myosotis-Honige an $M$ yos $)$ tisnektar berechnet sich bei Anwendung des Koeffizienten auf 0,95 p. roo. Praktisch genommen existieren also Vergissmeinnichthonige ïberhaupt nicht, denn schon eine kleine Beimengung von Vergissmeinnichtshonig führt zu einer sehr starken Einstäubung mit Myosotispollen. Was den Honig von Cynoglossum anbetrifft, der von I)r. Maurizlo zitiert wird, so enthält dieser-bei Anwendung der Pollenkoeffizienten - einen Anteil von 24 p. Ioo aus Cynoglossumtracht.

Die rumänischen Tilia-Honige, welche von Waben ohne Pollenzellen stammen, zeichnen sich durch einen geringen absoluten Pollengehalt aus. Er überschreitet nicht den Wert von 7 ooo Pollenkörner pro Io g Honig und betrug minimal 3000. Auch der absolute Gehalt an Tiliapollen ist gering; er schwankt zwischen $85^{\circ}$ und 4575 .

Die rumänischen und die sowjetischen Honige, die nach den üblichen Verfahren geerntet wurden, lassen sich in drei Gruppen unterteilen:

a) Honige mit geringem absolutem Gesamtpollengehalt, der durchschnittlich 5585 Pollenkörner in Io g beträgt. Auch der absolute Gehalt an 'Tiliapollen ist gering und beträgt durchschnittlich 91 2 Pollenkörner. Dies entspricht ungefähr der Hälfte des Tiliakoeffizientes. Der prozentuelle Anteil des Tiliapollens entspricht dem Einzelpollen;

b) Honige mit einem mittleren absoluten Gesamtpollengehalt (über I3300) und einem mittleren absoluten Gehalt an Tiliapollen (ca. 400o), der prozentuelle Anteil des 'Tiliapollens liegt zwischen Leitpollen und Begleitpollen;

c) Honige mit einem grossen absoluten Gesamtpollengehalt (ca. 35000) und 
grossen absoluten Gehalt an Tiliapollen (ïber I8 80o), der prozentuelle Anteil des Tiliapollen in dieser Gruppe liegt in den Grenzen Leitpollens.

Die rumänischen und die sowjetischen Honige stammen aus Stöcken ohne Aufsätze (20 Rahmen in Dadant-Mass). Fast alle Waben in den rumänischen Bienenstöcken enthielten Pollenzellen. Beim Entdeckeln und beim Schleudern gelangt

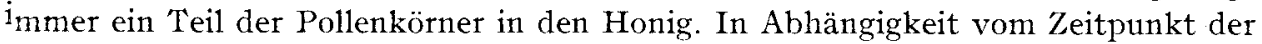
Honigernte überwiegt der Pollen der Pflanze, die gerade von den Bienen besucht wird.

Die Angaben bezüglich der Anzahl der Pollenkörner in den einzelnen Einartenhonigen aus verschiedenen Jahren weisen auf einen konstanten Einstäubungsgrad hin. Drei Honige aus Trifolium repens, die in drei Versuchsjahren gewonnen wurden, wiesen die folgenden Pollengehalte in je ro g auf: I6 510, I7 600 und I 7950 . Honig aus Melilotus albus enthielt 55060 (I959) bzw. 63700 (I956) Pollenkörner. Beinı Honig aus Rubus idaeus waren die Unterschiede grösser : 88020,56 I50 und 97400. Die nied rige Zahl 56 I 50 wurde durch den Schädling Byturus tomentosus verursacht, der die Bliiten beschädigte.

Die Einartenhonige von Malus, Rubus und Melilotus zeigten grosse Schwankungen im Pollengehalt innerhalb einer Versuchsperiode. I)ie erhaltenen Zahlen haben das Material für eine statistische Analyse geliefert. Bei $\mathrm{L}=0,05$ wurde festgestellt, dass die Zahl der Pollenkörner in I g Malus-Honig zwischen I 644 und 2534 (Versuchsjahr r956) schwankt, in Melilotus-Honig zwischen 5150 und 7590 und in Rubus-Honig zwischen 6588 und I I 032 . Der Rubus-Honig des Versuchsjahres I957 enthielt zwischen 9013 und I0464 Pollenkörner prog. Es zeigte sich, dass mit dem fortschreitenden Abblühen der Rubus-Versuchsparzelle der Pollengehalt des eingetragenen Honigs abnimmt. Dies ist wahrscheinlich dadurch bedingt, dass die Nektarsekretion länger anhält (mehrere Tage) als die Verstäubung des Pollens, die sich zu Beginn der Blüte vollzieht. Infolgedessen weist der während der späteren Blühstadien gesammelte Nektar und folglich auch der daraus gebildete Honig, einen niedrigeren Pollengehalt auf.

Auch im Melilotus-Honig wurden sehr starke Schwankungen festgestellt (zwischen ca. I 500 und 2500 Pollenkörner pro g) und dies sowohl im Bereich verschiedener Schichten der gleichen Zelle, wie auch zwischen Nachbarzellen der Wabe. Ier Honig-Einstäubungsgrad ist also ein Mittelwert von ganzer Vegetationsperiode.

Das hier zusammengestellte grosse Versuchsmaterial erlaubt es, den Wert der bisherigen Methode der Pollenanalyse in Frage zu stellen und ermöglicht gleichzeitig die Ausarbeitung einer neuen Methode, die auf den Pollenkoeffizienten basiert. Alle experimentell gewonnenen Honige wurden auf Grund des Finstäubungsgrades in 8 Klassen eingeteilt (s. tab. 2):

Bei der Aufstellung der Klassen wurde so verfahren, dass jede folgende Klasse Honige enthält, bei denen der Mittelwert der Pollenkoeffizienten um das doppelte so gross ist wie der der vorhergehenden Klasse. In gleicher Weise wird mit den Intervallen verfahren.

Die Mehrzahl der untersuchten Honige liegt in den Klassen 2, 4, 5 und 6. Die Klasse 2 (mit dem Koeffizienten 225) enthält 9 Arten ; die Klassen 4, 5 und 6 je 6 Arten. Von den Klassen Io bis 18 ist nur die Klasse I3 mit Cynoglossum officinale und die Klasse I 8 mit Myosotis silvatica besetzt.

Die genaue Beschreibung der Methode und die Art, wie man sich der Pollenkoeffizienten bedient, habe ich in meiner Arbeit : "Pollenkoeffizientien als Grundlage det quantitativen Pollenanalyse des Honigs " gegeben. 
TABELLE 2 TABLEAC 2

Klassenkoeffisienten der 46 linartenhonige Coefticients de classes des $4^{6}$ miels unifloraux

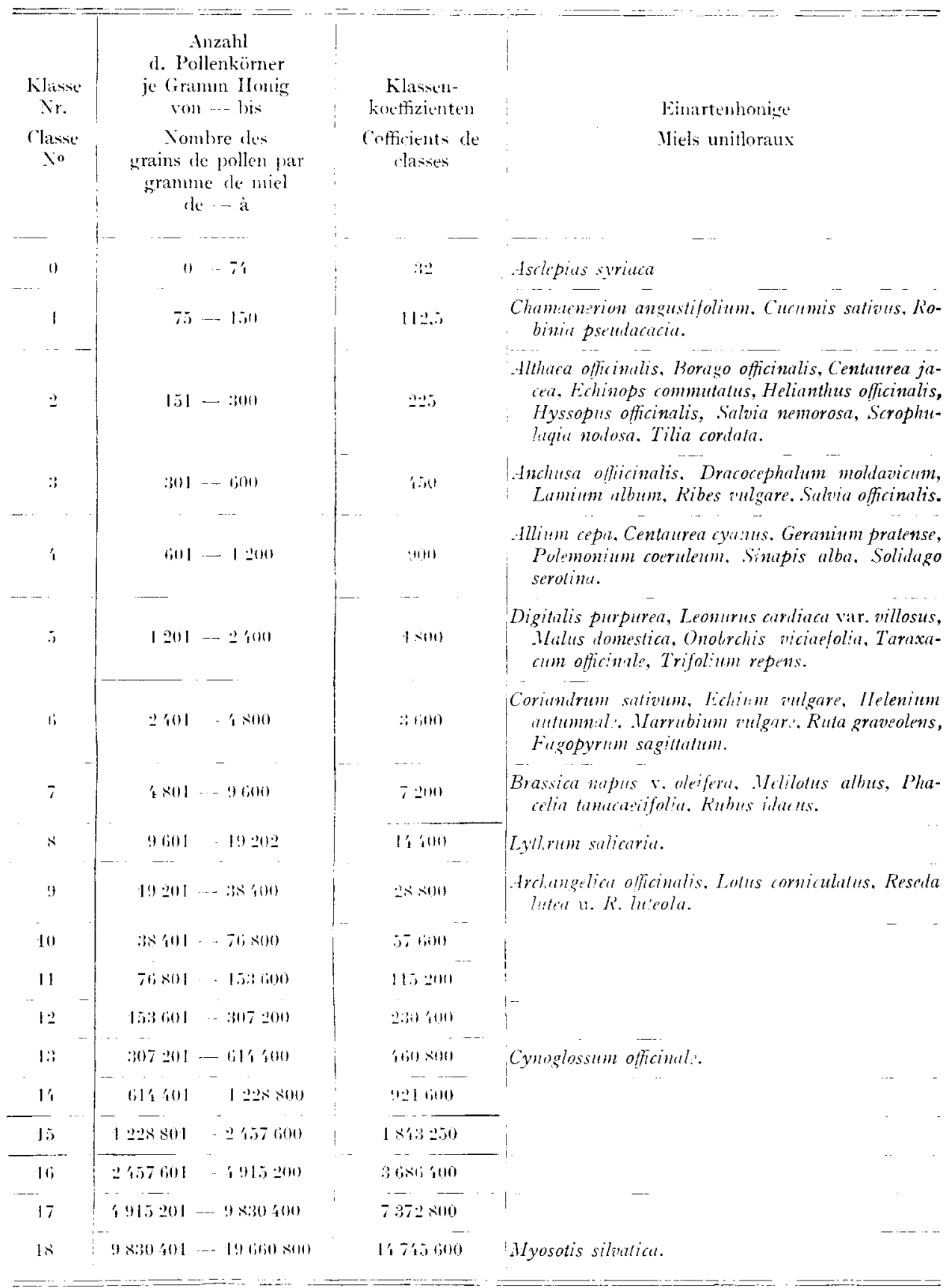


Auf dem Diagramm (Abb. x) wird der Zusammenhang zwischen der Koeffizienten. klasse (d. h. dem Grad der Einstäubung der Einartenhonige) und dem kleineren Durchmesser der Pollenkörner dargestellt. Es stellte sich heraus, dass sich die Honige auf eine eigenartige Weise gruppiert haben. In den höchsten Klassen finden sich die Honige, deren Pollen das kleinste Volumen haben. Umgekehrt finden sich in den niedrigsten Klassen die Honige mit grossen Pollenkörnern. Die Mehrzahl der Honige enthält Pollenkörner, deren kleinerer Durchmesser zwischen 20-25 $\mu$ beträgt ; diese Honige gehören den verschiedensten Klassen an. Dieses Phänomen lässt sich meiner Meinung nach durch zwei Faktoren erklären : einmal durch die Grösse der Pollenkörner, die den Grad der Honigreinigung durch die Bienen bestimmt; zum anderen durch den Bau der Blïte, die Zahl der Staubblätter und die Menge des Blïtenstaubs.

Auch die Intensität der Nektarproduktion beeinflusst den Einstäubungsgrad. Ist die Nektarproduktion sehr gross, dann wird der Nektar in geringerem Masse durch den Ventiltrichter der Bienen bearbeitet, daher ist seine "natürliche " Eins-

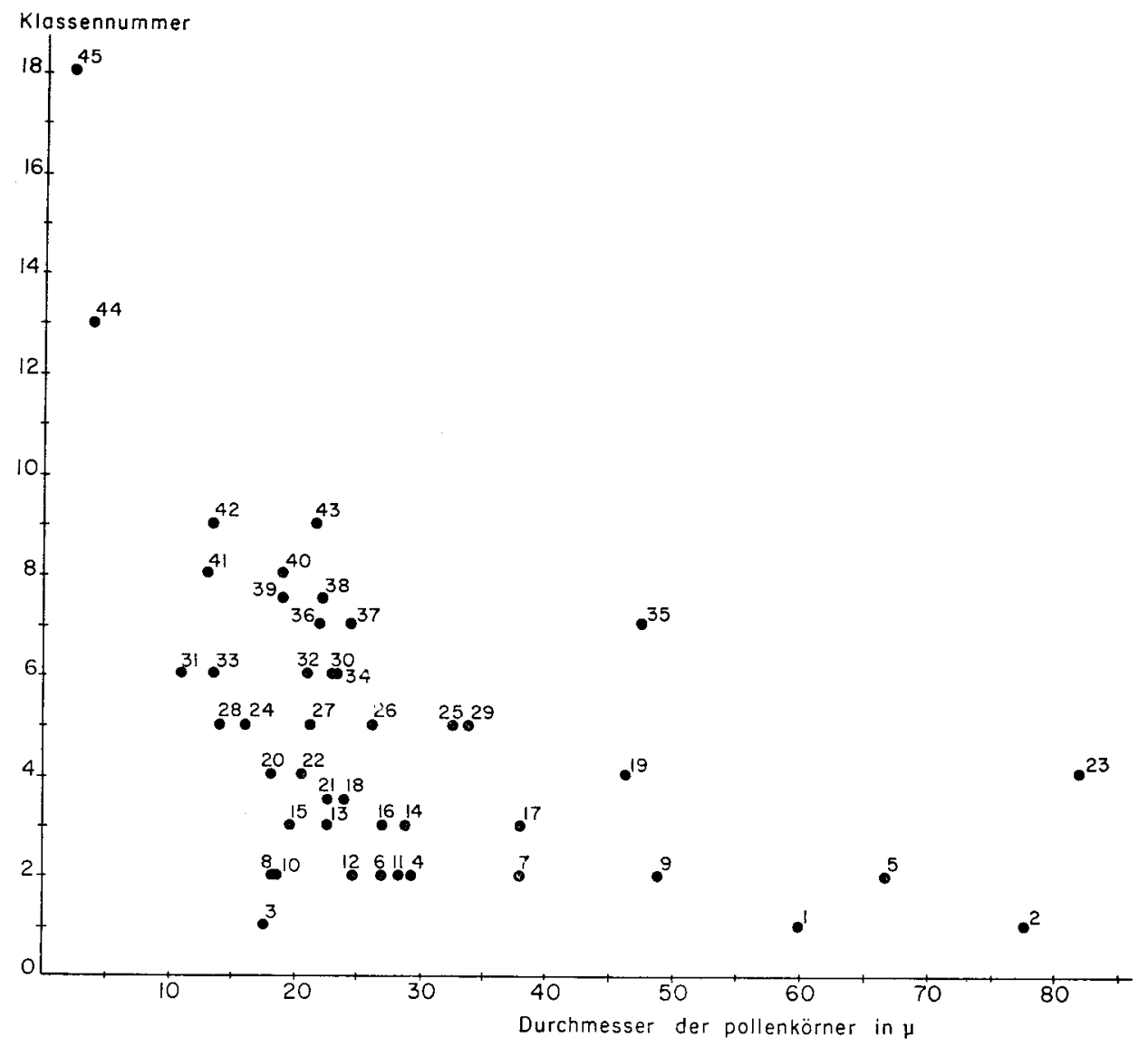

Aвв. I. - Zusammenhang zwischen dem Grad der Einstäubung der Einartenhonige und dem kleineren Durchmesser der Pollenkörner

Fig. I Rapport entre la richesse en pollen des miels aniforanx et le plus petit diamètre des grains de pollen 


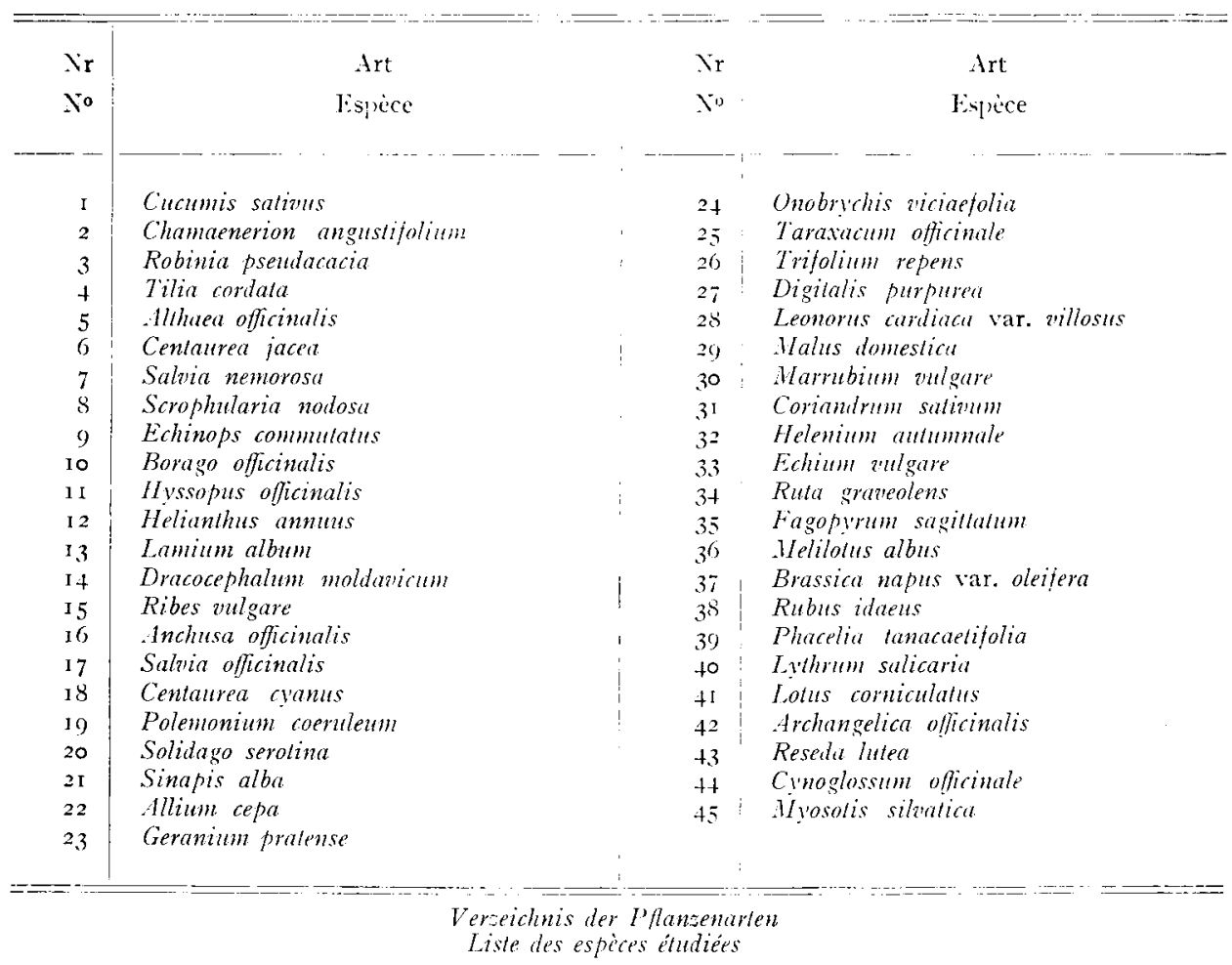

täubung grösser. Dagegen führt eine geringe Nektarproduktion zu einer intensiveren Bearbeitung des Nektars, und der entstehende Honig hat einen geringeren Einstäubungsgrad. Es gelang mir diese Hypothese experimentell zu untermauern. Auf zwei isolierten Feldchen mit einer bestimmten Versuchspflanze (Phacelia tanacaetifolia, Fagopyrum saggitatum, und Brassica napus var. oleifera) stellte ich zwei kleine Bienenvölkchen gleicher Grösse, aber verschidenener Volkszusammensetzung ; eines mit einer grossen Zahl von Sammlerinnen, eines mit einer geringen Zahl von Sammlerinnen. Im Falle von Phacelia sammelte das erste Versuchsvölkchen Honig mit einem Einstäubungsgrad von 8892 Pollenkörner/g. Der Honig des zweiten Völkchens enthielt nur I 249 Pollenkörner pro g, also ca. 7 mal weniger. Beim Versuch mit Brassica napus waren bei starkem Zufluss von Nektar in I g Honig I5 233 Pollenkörner nachzuweisen, bei mittlerem Zufluss 7960 und bei kleinem Zufluss I 592 .

Den Zusammenhang zwischen dem Finstäubungsgrad und der Intensität des Nektarzuflusses zum Stock konnte ich auch sonst beim Entnehmen der Raps und Buchweizenhonigen, die ich während der Trachtdauer direkt von Waben enthielte, beobachten. Deshalb hat man in manchen Fällen eine Korrektur der Koeffizienten vorzunehmen, so z. B. in Ländern, in denen man mit einer guten Trachtpflanze grosse Gebiete bebaut (z. B. die Sonnenblumenfelder in Rumänien).

Für die Finführung der Pollenkoeffizienten in die Pollenanalyse sprechen folgende Argumente : 


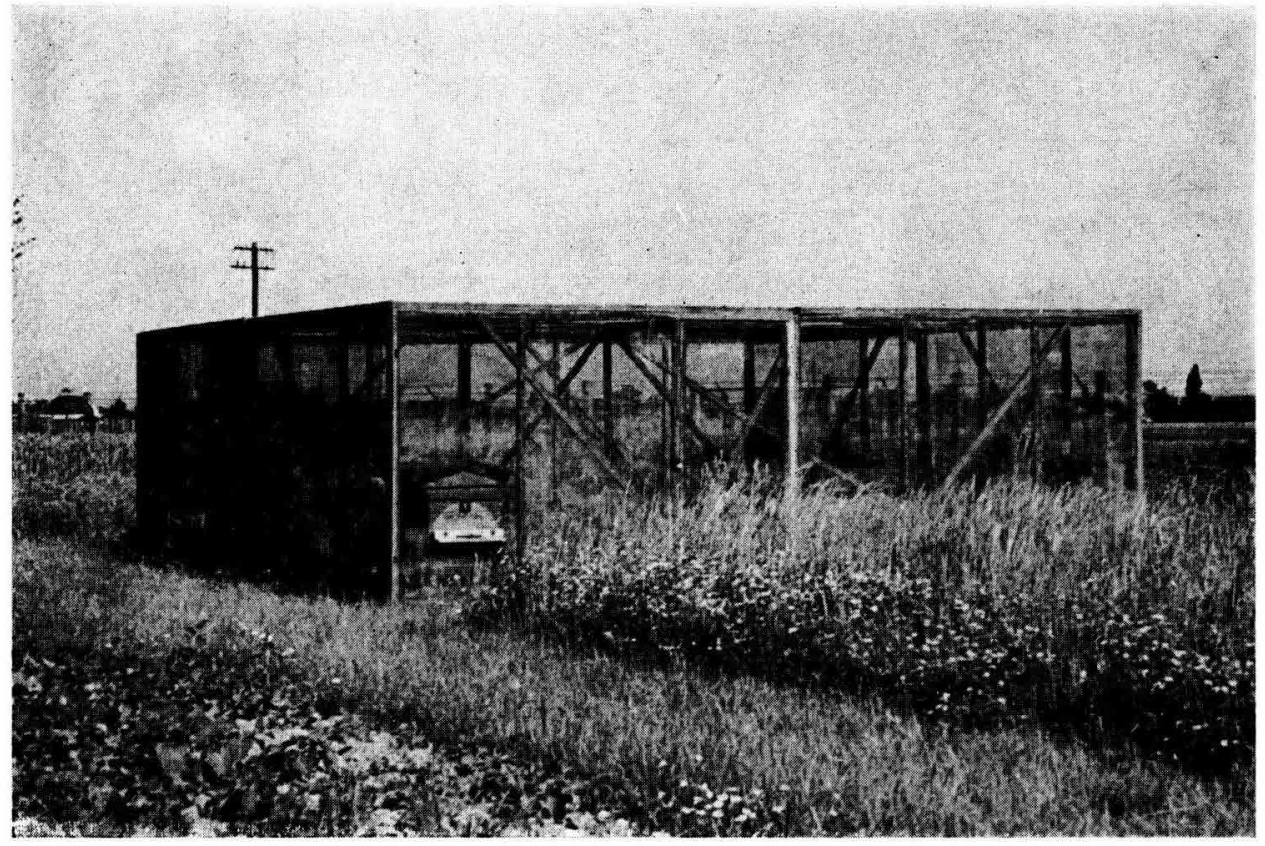

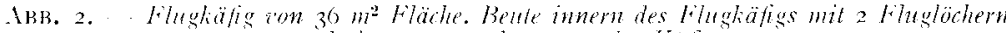
nach inmon the nach alusen des Katles

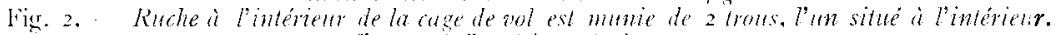
l'autre à l'extérieur de la cage.

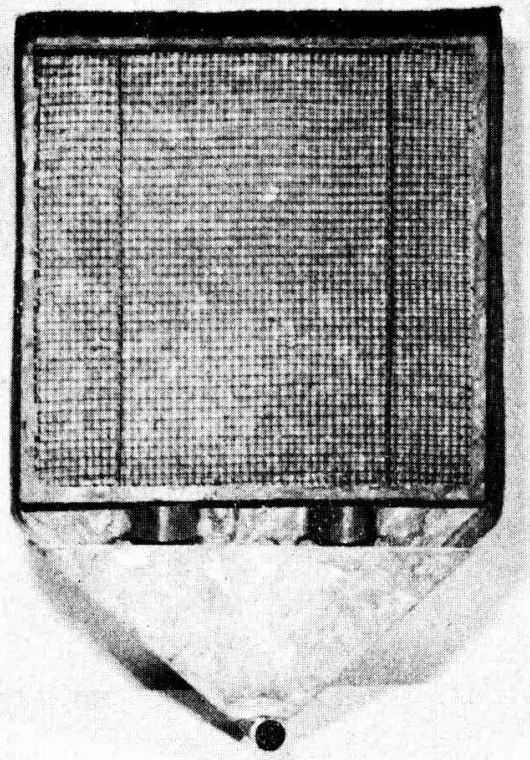

1вн. 3. Lecre Schlewdercinlage

Fis. 3. Exwroteur anite 


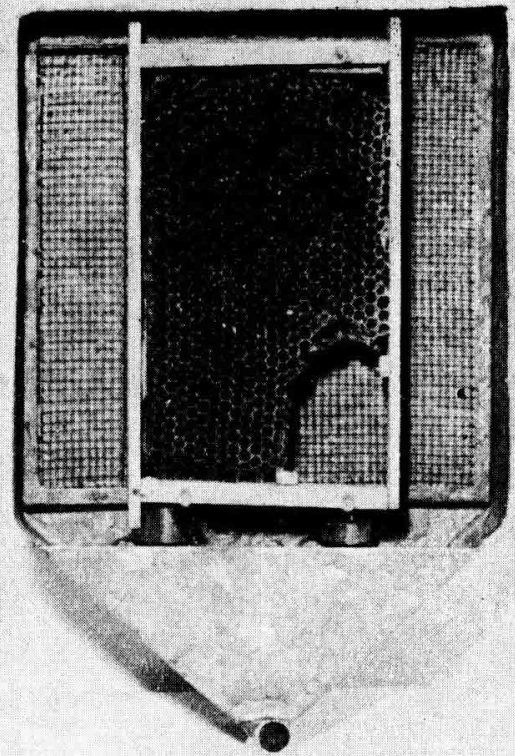

ABB. 4. - Einlage mil kleiner Wabe sum Schlendern vorgebildet l*ig. +. - Dispositif avec un rayon préparé pour l'extraction

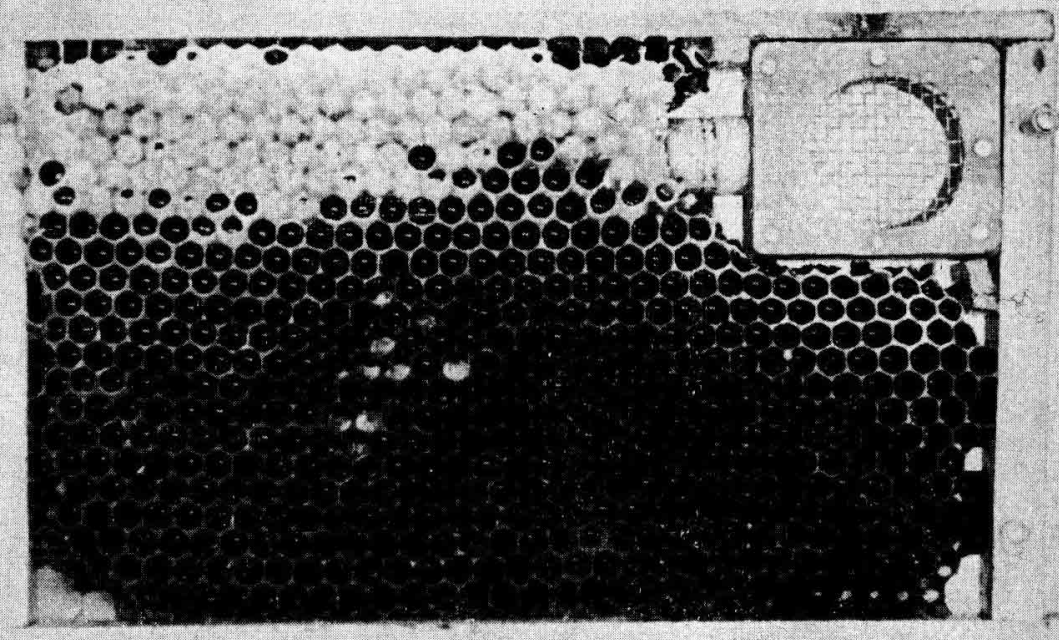

Aвв. 5. - Wäbchen mil Honig und Pollen von Melilotus

Fig. 5. - Ravon avec miel et pollen de Yelilotus 
I. Die grosse Strenung des Einstäubungsgrades in Einartenhonigen von $\alpha$ - I73 ogo ooo Pollenkörnern je Io g Honig. Die Herabsetzung der Grenze für den Leitpollen auf IO-I5 p. IOo nützt nicht viel, weil die Unterschiede zwischen den Klassenkoeffizienten benachbarter Klassen roo p. Ioo betragen und für jede folgende noch entsprechend grösser sind.

2. Die Methode erlaubt die richtige Feststellung der Trachtanteile im Honig, besonders der Trachtanteile, die natürlicherweise pollenarm sind und daher nach dem bisherigen Verfahren unterbewertet wurden, weil die entsprechenden Pollen nur als Einzelpollen » auftraten.

3. Mit Hilfe der vorgeschlagenen Methode können wir uns auch vergewissern, ob der Honig sekundär eingestäubt ist.

\title{
RÉSUMÉ
}

\author{
CARACTÉRISTIQUES DES MIELS UNIFLORAUX
}

L'analyse pollinique des miels repose sur le fait que les abeilles butineuses de nectar récoltent: avec chaque gouttelette de cette substance un certain nombre de grains de pollen provenant de la fleur qui a été visitée. Ce pollen reste ensuite dans le miel dérivé du nectar; son identification permet dans une large mesure de reconnaître l'origine botanique du miel. Il convient cependant de noter que la richesse en pollen des différents miels est très variable. Les différences sont en rapport avec les phases de la floraison, la morphologie de la fleur qui a fourni le nectar ainsi qu'avec de nombreux facteurs. 11 a donc paru intéressant de rechercher pour différentes plantes mellifères dans quelle marge se situe la richesse en pollen des miels auxquels elles donnent naissance.

La méthode mise au point par l'auteur consiste à faire récolter sous des cages (fig. 2) de grandes. dimensions $\left(36\right.$ a $64 \mathrm{~m}^{2}$ ) et par de très petites colonies d'abeilles (300 à 400 abeilles avec une reine). des miels rigoureusement purs provenant des cultures disposées sous lesdites cages. On obtient de. la sorte de petites quantités de miel (de quelques grammes jusqu'à $35^{\circ} \mathrm{g}$ ) que l'on peut qualifier d'unifloraux. L'analyse pollinique quantitative de ces miels permet ensuite de connaître avec une grande précision leur teneur absolue en pollen.

Les tableaux $\mathrm{x}$ et 2 constituent le résumé de l'ensemble des données qui ont été acquises au cours. des 13 dernières années en utilisant la méthode ci-dessus décrite. Au total, 46 espèces végétales ont été étudiées; elles appartiennent à 22 familles différentes. La teneur absolue en pollen est très. variable puisqu'elle va de o (Asclepias syraca) jusqu'ì $\mathrm{I} 73090$ ooo (Myosotis silvatica) pour Io $\mathrm{g}$. de miel. Toutefois, pour un très grand nombre d'espèces la teneur en pollen se situe autour de quelques dizaines de milliers par Io g. Par ailleurs, on peut noter qu'il existe une certaine corrélation entre la richesse en pollen et la taille des grains : en règle générale, plus le pollen est petit plus le miel est riche (fig. I). De même l'intensité de l'afflux du nectar dans la colonie exerce une influence considérable sur le degré de la richesse en pollen, ce que l'auteur a réussi à prouver à l'aide des expériences effectuées. I.orsque l'afflux de nectar est important la richesse en pollen est grande, par contre, lorsqu'il est faible, la teneur en pollen est faible également.

Se servant des données obtenues expérimentalement, l'auteur répartit les différents miels unifloraux en i 8 classes successives, chaque classe étant caractérisée par une richesse moyenne deux fois plus grande que la classe précédente. Chaque classe se trouve affectée d'un coefficient qui doit être utilisé dans les analyses polliniques en vue de redresser les spectres obtenus par dénombrement des grains de pollen et de corriger ainsi les erreurs qui pourraient être commises dans l'interprétation des spectres polliniques lorsqu'on recherche l'origine botanique des miels. La méthode est particulièrement utile pour évaluer l'importance rélle comme source de nectar des plantes dont le nectar est, soit très riche, soit très pauvre en pollen. En même temps elle permet de vérifier si la richesse en pollen du miel étudié n'est pas secondaire. 
SUMMARY

CHARACTERISTICS OF UNIFLOROUS HONEYS

The pollen analysis of honevs is based on the fact that nectar-gathering bees collect, with each rroplet of this substance, a certain number of grains of pollen from the visited flower. This pollen then finds itself in nectur honey; its identification allows to a large extent the determination of the botanical origin of the honey. It should be remarked, however, that the richness of the pollen from different honeys varies a great deal. The differences are related to the morphology of the flower from which the nectar is extracted, and also numerous other factors. It is therefore considered expedient to determine for the various honeys the content in pollens from various melliferous plants.

The method devised by the author consists in gathering honey under large cages (fig. 2) (36 to $64 \mathrm{~m}^{2}$ ) and by very very small colonies of bees (300 to 400 bees with one queen) rigorously pure honey from cultures housed in these cages. Simall quantities of honey (from just a few to $350 \mathrm{~g}$ ) are thus obtained which can be classified as uniflorous honey. The quantitative pollen analysis of these honeys gives a more accurate picture of the absolute pollen content.

Tables $\mathrm{I}$ and 2 summarise the data gathered over the last i 3 years using the method described above. In all, 46 vegetal species have been studied; they belong to 22 different families. 'The absolute pollen content varies very considerably, ranging from o (Asclepias syriaca) up to $173,090,000$ ( $\mathrm{Myo}$ sotis sylvatica) per $10 \mathrm{~g}$ of honey. Nervertheless, for a very large number of species, the pollen content was around a few tems of thousands per $10 \mathrm{~g}$. Furthermore, there is an apparent relationship between the richness in pollen and the size of the grains : as a general rule, the smaller the pollen, the richer the honey (fig. I).

Tsing the experimental data, the author arranged the different uniflorous honevs in I 8 successive groups, each group having an average richness two times greater than the preceding group. Lach group has a coefficient which can be used in pollen analyses in order to check the varieties identified by counting the pollen grains, and thus to correct any inajor errors which could have occurred in the interpretation of the pollen varieties in trying to determine the botanical origin of honeys. This method is particularly helpful in evaluating the true importance as a nectar source of plants whose nectar is either very rich or very poor in pollen.

\section{RÉFÉRENCES BIBLIOGRAPHIQUES}

Barbier E., i958. Examen pollinique de quelques miels unifloraux. Am Abeille, 1, 73-76.

Barbier li. et Pangauj) (Cl., Ig6r. Origine botanique et caractéristiques physicochimiques des miels. Ann. Abeille, 4, 51-65.

Berner U., 1952. Die Auswertung der P'ollenanalyse. Arch. Bienenkunde, 29, 33-38.

Beutler R., Wahl O., i938. Über das Honigen der Linde in Deutschland. Z. vergleich. Physiol., 23 (3), $301-33 \mathrm{I}$.

Demianowicz A., Deminnowicz Z., ${ }^{1955 .}$ A new method of pollen analysis of honeys. Prace Instytuty Sadownictwa, 1, 185-195 (Polnisch mit englischer Zusammenfassung).

Demianowicz A., Demianowicz Z., 1956. Neue Grundlagen der Pollenanialyse des Honigs. XVI Intern. Bienenzüchterkongress. Wien. Prot. $11^{\circ} 395$.

Demianowicz A., Demianowicz Z., 1957. A new method of pollen analysis of honeys. Pszczel. Zesz. Vauk., 1 (2), 69-78 (Polnisch mit englischer Zusammenfassung).

Demranowicz Z., I960. Problem der Vergissmeinnichthonige. Pszcsel. Zess. Yauk., 4 (1), $+3-48$ (Polnisch mit deutscher Zusammenfassung).

Deminowicz Z., 196r. Pollenkoeffzienten als Grundlage der quantitativen Pollenanalyse des Honigs. Pszczel. Zesz. Nauk., 5 (2), 95-105.

Demianowicz Z., 1962 a. Die Beurteilung der Ënsortenlindenhonige bei der Anwendung der Pollenkoeffizientenmethode. Pszcsel. Zesz. Sauk., 6, (1), 25-45 (Polnisch mit deutscher Zusammenfassung).

Demaniowicz Z., I I 62 b. Some features of one species honey obtained from 6 plants of Labiatae family. Pszczel. Zesz. Nauk., 6 (2), 75-8o (Polnisch mit englischer Zusammenfassung). 
Demasowicz Z., 1963. Das Pollenspektrum des Honigs beeinflussende Faktoren. XIX Intern. Bienenziichterkongress. Prag., 33-3+.

Deniaxowicz Z., Denilyowicz 1., 1952. Prüfung der Feststellung des Pollenkoeffizients des Senfhonig (Sinapis alba L.). Billelln $\operatorname{Ir} \mathbf{2}(4)$ CIR. (Polnisch).

Deniaxowicz Z., HIאx II., 196o. Vergleichuntersuchungen der Nektarsekretion bei 17 Lindenarten. Pszcsel. Zesz. Vauk., 4 (3-t), 133-152 (Pohnisch mit deutscher Zusammenfassung).

Demianowicz, Z., Jablowsil B., I959. Über die Pollenkoeffizienten von 3 sortenreinen IIonigen vom Standpunkt der statistischen Analyse. Psiczel. Zess. Nauk., 3 (1), 25-34) (Polnisch mit deutscher Zusammenfassungr).

Louveaux J., I956. Étude des miels français par l'unalyse pollinique. XVI Intern. Bienensïchterkongress., Wien. Prot. $\operatorname{Nr} 637$.

Louveaux J., I958. Recherches sur l'origine dans le miel du pollen de plantes entomophiles dépourvues de nectaires. Inn. Abeille, 2, 89-92.

MAERz A., PALL M., I950. A dictionary of color.

Macrizio A., 1936. Gibt es Lindenhonig in der Schweiz? Schweiz. Bienenstg. (I2).

Malrizio A., I939. Lintersuchungen zur quantitativen Pollenanalyse des Ionigs. Trat. chim. aliment. hyg., Suisse, $30(1-2), 1-45$.

Maurizio A., 1940. Schweiz. Honigtypen. 3. Vergissmeinnichthonig. Schweis. Bienenstg.

Maurizio A., I949. Pollenanalytische Untersuchungen an Honig und Pollenhöschen. Schaeiz. Bienenstg., $2(18), 319-455$.

Malrizio A., i955. Beiträge zur quantitativen Pollenanalyse des Honigs. Z. Bienentorsch., 3 (2), $32-39$.

PrITsch G., 195\%. Zum Problem der mikroskopischen Pollenanalyse des Honigs. Wissensch. Z. HumboldtUniversitäl, $6(2), 9 i^{-204}$.

ZANDER F., 1935. Beiträge zur Herkunftbestimmung bei Honig. Pollengestaltung und Herkunftbestimmung bei Blütenhonig. Bd. I u. II. 